\title{
Capacitação dos profissionais de saúde e seu impacto na detecção precoce do câncer de mama
}

\author{
Training of health professionals and its impact on the early detection of breast cancer
}

\author{
Formación de los profesionales sanitarios y su impacto en la detección precoz del cáncer \\ de mama
}

Ramon Veloso Sousa Sobral ${ }^{1 *}$, Nathan Henrick Sirqueira Kretli ${ }^{1}$, Rosiane Luz Cavalcante ${ }^{1}$, Janete de Oliveira Briana1, Daniela Batista Ferro', Helane Conceição Damasceno.

\section{RESUMO}

Objetivo: Avaliar o rastreamento de Câncer de Mama por meio da solicitação de mamografia. Métodos: Trata-se de uma pesquisa quantitativa, aplicada, exploratória e de levantamento de dados secundários referentes à solicitação de mamografias, na base de dados do Sistema de Informação DATASUS, entre os períodos de julho/2018 a julho/2020 em intervalos de pré e pós capacitação aplicada na Região de Integração do Xingu, localizada na região norte do Brasil, Estado do Pará. Resultados: A mamografia de rastreio fator idade (40 a 49 anos) na região de integração do Xingu obteve diminuição das solicitações na ordem de 634 para 350. Destaca-se também a disparidade de solicitações para mamografia de rastreio (1.108) diante das solicitações para mamografia diagnóstica (12). Conclusão: Houve uma diminuição significativa no número de mamografias de rastreio baseadas na população alvo por idade, ressaltando o aumento percentual no número de mamografias de rastreio nas idades preconizadas pelo Ministério da Saúde e diminuição nas demais idades. Os resultados alcançados foram positivos e geraram efeitos, porém limitados. Dessa forma, reforçando a necessidade de uma educação permanente aos profissionais da região acerca do tema.

Palavras-chave: Câncer de Mama, Diagnóstico precoce, Atenção primária à saúde.

\section{ABSTRACT}

Objective: To evaluate the screening for Breast Cancer through the request for mammography. Methods: This is a quantitative, applied, exploratory research and survey of secondary data recurring to the request for mammograms, in the database of the DATASUS Information System, between the periods of July / 2018 to July / 2020 at intervals of pre and post training applied in the Xingu Integration Region, located in the northern region of Brazil, State of Pará. Results: The screening mammography factor age (40 to 49 years) in the Xingu integration region received a request in the order of 634 for 350 . There is also a persistence disparity for screening mammography $(1,108)$ in face of requests for diagnostic mammography (12). Conclusion: There was a decrease in the number of screening mammograms based on the target population by age, highlighting the percentage increase in the number of screening mammograms at the ages recommended by the Ministry of Health and a decrease at other ages. The results achieved were positive and effects generated, but included. Thus, reinforcing the need for continuing education for professionals in the region on the subject.

Key words: Breast cancer, Early diagnosis, Primary health care.

\section{RESUMEN}

Objetivo: evaluar el cribado de cáncer de mama a través de la solicitud de mamografía. Métodos: Se trata de una investigación y levantamiento cuantitativo, aplicado, exploratorio de datos secundarios recurrentes a la solicitud de mamografías, en la base de datos del Sistema de Información DATASUS, entre los períodos de julio 2018 a julio 2020 en intervalos de pre y post entrenamiento aplicada en la Región de Integración del Xingu, ubicada en la región norte de Brasil, Estado de Pará. Resultados: Mamografia de rastreamento para criança de um ano (40 a 49 anos) na região de integração do Xingu obtém redução de solicitações de 634 para 350. Hay también una disparidad persistente para la mamografía de detección $(1,108)$ frente a las solicitudes de mamografía de diagnóstico (12). Conclusión: Hubo una disminución significativa en el número de mamografías de detección según la población objetivo por edad, destacando el aumento porcentual en la

${ }^{1}$ Universidade Federal do Pará (UFPA), Altamira - PA. *E-mail: ramon.sobral@altamira.ufpa.br 
cantidad de mamografías de detección en las edades recomendadas por el Ministerio de Salud y una disminución en otras edades. Los resultados obtenidos fueron positivos, generando efectos, pero limitados. De ahí que se necesite una educación permanente a los profesionales de la región sobre el tema.

Palabras clave: Cáncer de mama, Diagnóstico precoz, Atención primaria de salud.

\section{INTRODUÇÃO}

Conforme Jesinger RA (2014), conhecer anatomia da mama feminina é imprescindível ao planejamento de intervenções seguras, assim como é de suma importância no reconhecimento de complicações indesejadas em determinados procedimentos. Esta pode ser avaliada em diferentes perspectivas e uma delas é por intermédio dos variados exames de imagem.

Entender os padrões anatômicos das mamas ajudam os intervencionistas reconhecerem as fisiopatologias, dentre estas destaca-se o câncer de mama (JESINGER RA, 2014). Visto que esta doença possui alta prevalência a qual incide as mulheres no Brasil, excetuando-se o câncer de pele não melanoma (INCA, 2015).

As estruturas anatômicas essenciais da mama feminina são: pele, gordura, camadas fasciais, ligamentos de cooper, tecido fibroglandular, vasos linfáticos e estruturas neurovasculares; todas essas estão posicionadas sobre a parede torácica (BRASIL, 2013). O principal suprimento de sangue arterial deriva da artéria torácica interna; anatomia venenosa e drenagem linfática geralmente são paralelas à anatomia arterial, com variações nos canais de comunicação entre os canais venosos e linfáticos profundos e superficiais (BRASIL, 2014).

A eficácia na detecção precoce do câncer de mama baseia-se em: Exame Clínico de Mamas (ECM) e a Mamografia (BRASIL, 2015). Uma vez que o ECM é a parte fundamental da propedêutica para o diagnóstico de câncer. De acordo com o controle do câncer de mama, em seu documento de consenso de 2014, o ECM deve fazer parte do atendimento integral de todas as mulheres no exame físico e ginecológico, de forma independente da faixa etária, sendo constituinte básico para solicitação de exames complementares (BRASIL, 2014). Em razão disso, nota-se que o exame físico contribui para construção clínica favorecendo, portanto, para um bom diagnóstico (SILVA C, et al., 2009)

No mundo, o câncer de mama é uma mácula humana e uma causa de mortalidade prematura entre as mulheres. Diversos fatores de risco para câncer de mama foram bem estabelecidos por estudos epidemiológicos, incluindo etnia, raça, características genéticas e história familiar de câncer, bem como exposições modificáveis, a exemplo do aumento do consumo bebidas alcoólicas, sedentarismo, hormônios exógenos e primeiro filho após os 35 anos (COUGHLIN SS e CYPEL Y, 2019).

É válido ressaltar que, de forma universal, independentemente da condição de desenvolvimento econômico, a mama é o local mais frequente de câncer nas mulheres (GARNIER HS e COLONNA M, 2019). Verificou-se uma estimativa de 18,1 milhões de novos casos de câncer (17 milhões excluindo câncer de pele não melanoma) e 9,6 milhões de mortes por câncer (9,5 milhões excluindo câncer de pele não melanoma) em todo o mundo em 2018 (FERLAY J, et al., 2018). Estimativa esta superior ao último levantamento, onde 14,1 milhões de antigos casos e 8,2 milhões de mortes em 2012, sendo esta última 1,67 milhão ocasionado por mama (FERLAY J, et al., 2018).

A estimativa de Incidência de Câncer no Brasil (INCA, 2019), válida ao ano de 2020, prevê 223.110 novos casos correspondente a todas as Neoplasias, exceto pele não melanoma, nas mulheres brasileiras. Considerando o triênio 2020-2022, é estimado que 66.280 (29,7\%) atuais casos de câncer de mama. Por conseguinte, resultando em risco de 61,61 casos novos para cada 100 mil mulheres anual.

Assim, é público que o câncer de mama feminino, desconsiderando o câncer de pele não melanoma, ocupa a primeira posição no ranking mais frequentes em todas as cinco macrorregiões. Na Região Norte 0 risco estimado é de 21,34 por 100 mil. No Pará o risco estimado é de 18,24 por 100 mil, bem como no Estado temos setecentos e oitenta (780) casos novos de Câncer de Mama, destes quatrocentos e sessenta (460) são dos demais municípios excetuando-se a Região Metropolitana de Belém (BRASIL, 2019). 
Por conseguinte, partindo da importância do assunto para o âmbito da atenção básica na Região de Integração do Xingu, é de suma importância que os profissionais da saúde possam entender e diferenciar "rastreamento" de "investigação", bem como compreender o papel da atenção básica no assunto. Assim como, é imprescindível para estes profissionais a compreensão das diretrizes brasileira de rastreamento do câncer de mama no quesito da solicitação da mamografia de rastreio a qual inclui as subdivisões: "rastreio população alvo, população de risco elevado e população já tratada, assim, como a solicitação da mamografia de diagnóstico", visando otimizar a realização do exame pela mulher e reduzir a sobrecarga de indicações desnecessárias do referido exame.

\section{MÉTODOS}

O presente estudo trata-se de uma pesquisa quantitativa, aplicada, exploratória e de levantamento de dados secundários referentes à solicitação de mamografias de rastreio e diagnósticas no Estado do Pará na Região de Integração do Xingu (Altamira, Anapu, Brasil Novo, Medicilândia, Pacajá, Porto de Moz, Senador José Porfírio, Uruará e Vitória do Xingu), utilizando a plataforma do DataSUS, com tabulação de dados pela ferramenta Tabwin - SISCAN. Este estudo segue a resolução 510/2016 do Conselho Nacional de Saúde que dispensa a avaliação pelo Comitê de Ética em Pesquisa por não identificar o participante da pesquisa.

Foi realizada atividades de educação permanente nos 9 municípios da região Xingu entre os meses de junho e julho de 2019, com intuito de atualizar os profissionais médicos, enfermeiros e profissionais da central de regulação municipal acerca da indicação de solicitação de exames de mamografia de rastreamento e mamografia de diagnóstico seguindo as diretrizes Brasileiras de rastreamento do câncer de mama preconizado pelo MS.

De acordo com Ministério da saúde a indicação clínica para solicitação da mamografia pode ser: mamografia de rastreamento ou mamografia de diagnóstico. As mamografias de rastreamento são divididas em três formas de indicação: rastreamento de população alvo por idade, população de risco elevado (que possui histórico familiar de primeiro grau de câncer de mama ou de ovário) e pacientes já tratados de câncer de mama. Quando o paciente já possui sinais clínicos que necessitam do exame de imagem, a mamografia solicitada deve ser uma mamografia diagnóstica.

A partir da conclusão do processo de educação em permanente em saúde foram pesquisados todos os dados de solicitação de mamografias em dois períodos diferentes: 1ำ momento de junho de 2018 a junho de 2019, período antecedente às oficinas de atualização e segundo momento de julho de 2019 a julho de 2020, período posterior as oficinas. As informações foram consolidadas considerando os municípios em que as oficinas foram realizadas, a partir de então procedeu-se a coleta a coleta de dados no mês de setembro de 2020 com intuito de comparar os dados obtidos no primeiro e segundo momento.

Foram incluídos todos os resultados de exames de mamografia disponíveis no período da pesquisa no sistema DATASUS (SISCAN). Incluindo na tabulação: Local de solicitação do exame, Município da Unidade de Saúde, Indicação clínica e tipo de mamografia de rastreamento. O critério de exclusão inclui: mamografias solicitadas fora das localidades de estudo. Os dados coletados foram processados utilizando o software Microsoft Office Excel 2010 e analisados através da estatística descritiva.

\section{RESULTADOS}

Destaca-se a solicitação de mamografia da região Xingu detalhada por município de solicitação no período pré e pós capacitação evidencia os dados gerais. Levando-se em consideração a Região Xingu, no período anterior as oficinas foram registradas 1778 mamografias solicitadas, sendo que deste total, $1726(97 \%)$ foram mamografias de rastreamento de população alvo, $31(1,7 \%)$ foram mamografias de rastreamento de risco elevado, $13(0,7 \%)$ mamografias de rastreamento de pacientes já tratadas e $8(0,4 \%)$ mamografias de diagnóstico.

No período posterior a realização das oficinas obteve-se os seguintes resultados: 1120 mamografias solicitas, sendo que destas, $1047(93,4 \%)$ foram mamografias de rastreamento de população alvo, 40 (3,57\%) foram mamografias de rastreamento de risco elevado, 21 (1,8\%) mamografias de rastreamento de pacientes já tratadas e 12 (1\%) mamografias de diagnóstico (Tabela 1). 
Tabela 1 - Avaliação da solicitação de mamografia nos Municípios de Realização da Atualização das Diretrizes de Rastreamento do Câncer de mama.

\begin{tabular}{|c|c|c|c|c|c|c|c|c|c|c|c|c|c|}
\hline \multirow{3}{*}{$\begin{array}{c}\begin{array}{c}\text { Municípios da Unidade } \\
\text { de Saúde }\end{array} \\
\text { (Região de Integração) }\end{array}$} & \multirow{3}{*}{ Período de Análise } & \multicolumn{11}{|c|}{ Mamografia de rastreamento } & \multirow{3}{*}{$\begin{array}{c}\text { Mamografia } \\
\text { Diagnóstica } \\
\text { Total }\end{array}$} \\
\hline & & \multicolumn{7}{|c|}{ População alvo por idade } & \multicolumn{3}{|c|}{$\begin{array}{c}\text { População de risco } \\
\text { elevado } \\
\text { (História Familiar) }\end{array}$} & \multirow{2}{*}{$\begin{array}{c}\text { Paciente } \\
\text { já tratado } \\
\text { de câncer } \\
\text { de mama }\end{array}$} & \\
\hline & & $\begin{array}{c}09 \text { a } \\
19\end{array}$ & $\begin{array}{c}20 a \\
29\end{array}$ & $\begin{array}{c}30 \mathrm{a} \\
39\end{array}$ & $\begin{array}{c}40 a \\
49\end{array}$ & $\begin{array}{c}50 \mathrm{a} \\
69\end{array}$ & $70+$ & Total & $\begin{array}{l}<35 \\
\text { anos }\end{array}$ & $\begin{array}{l}>35 \\
\text { anos }\end{array}$ & Total & & \\
\hline \multirow{2}{*}{ Altamira } & Pré-Capacitação & 0 & 7 & 52 & 385 & 584 & 38 & 1066 & 0 & 3 & 3 & 1 & 3 \\
\hline & Pós-Capacitação & 0 & 2 & 29 & 233 & 342 & 23 & 629 & 0 & 10 & 10 & 11 & 7 \\
\hline \multirow{2}{*}{ Anapu } & Pré-Capacitação & 0 & 0 & 2 & 36 & 42 & 6 & 86 & 0 & 0 & 0 & 2 & 4 \\
\hline & Pós-Capacitação & 0 & 0 & 1 & 20 & 29 & 0 & 50 & 0 & 3 & 3 & 1 & 3 \\
\hline \multirow{2}{*}{ Brasil Novo } & Pré-Capacitação & 0 & 0 & 2 & 39 & 54 & 2 & 97 & 1 & 25 & 26 & 4 & 1 \\
\hline & Pós-Capacitação & 0 & 0 & 0 & 21 & 77 & 3 & 101 & 0 & 4 & 4 & 1 & 2 \\
\hline \multirow{2}{*}{ Medicilandia } & Pré-Capacitação & 0 & 2 & 11 & 73 & 110 & 9 & 205 & 0 & 0 & 0 & 0 & 0 \\
\hline & Pós-Capacitação & 0 & 0 & 3 & 18 & 57 & 1 & 79 & 0 & 1 & 1 & 3 & 0 \\
\hline \multirow{2}{*}{ Pacaja } & Pré-Capacitação & 0 & 0 & 3 & 42 & 47 & 1 & 93 & 0 & 0 & 0 & 2 & 0 \\
\hline & Pós-Capacitação & 0 & 0 & 2 & 14 & 49 & 2 & 67 & 0 & 13 & 13 & 1 & 0 \\
\hline \multirow{2}{*}{ Porto de Moz } & Pré-Capacitação & 0 & 0 & 3 & 17 & 8 & 0 & 28 & 0 & 0 & 0 & 0 & 0 \\
\hline & Pós-Capacitação & 0 & 1 & 1 & 11 & 13 & 0 & 26 & 2 & 1 & 3 & 0 & 0 \\
\hline \multirow{2}{*}{ Senador Jose Porfírio } & Pré-Capacitação & 0 & 0 & 2 & 13 & 11 & 1 & 27 & 0 & 0 & 0 & 4 & 0 \\
\hline & Pós-Capacitação & 0 & 0 & 2 & 11 & 13 & 2 & 28 & 0 & 0 & 0 & 3 & 0 \\
\hline \multirow{2}{*}{ Uruara } & Pré-Capacitação & 0 & 0 & 5 & 22 & 44 & 0 & 71 & 0 & 2 & 2 & 0 & 0 \\
\hline & Pós-Capacitação & 0 & 0 & 0 & 14 & 33 & 1 & 48 & 0 & 6 & 6 & 1 & 0 \\
\hline \multirow{2}{*}{ Vitoria do Xingu } & Pré-Capacitação & 0 & 1 & 3 & 7 & 40 & 2 & 53 & 0 & 0 & 0 & 0 & 0 \\
\hline & Pós-Capacitação & 0 & 0 & 1 & 8 & 9 & 1 & 19 & 0 & 0 & 0 & 0 & 0 \\
\hline \multirow{2}{*}{$\begin{array}{c}\text { Região de Integração do } \\
\text { Xingu }\end{array}$} & Pré-Capacitação & 0 & 10 & 83 & 634 & 940 & 59 & 1726 & 1 & 30 & 31 & 13 & 8 \\
\hline & Pós-Capacitação & 0 & 3 & 39 & 350 & 622 & 33 & 1047 & 2 & 38 & 40 & 21 & 12 \\
\hline
\end{tabular}

Fonte: Sobral RVS, et al., 2020; dados extraídos do Sistema de Informação do Câncer (SISCAN). 
Evidencia-se desfecho da solicitação de mamografia de rastreamento população alvo por faixa etária no período anterior e posterior a realização das oficinas, observando que não houve solicitação de mamografias na faixa etária de 0 a 19 anos, nas idades entre 20 a 29 a solicitação anterior a capacitação foi $0,6 \%$ e posterior $0,3 \%$, entre 30 a 39 anos teve-se $4,8 \%$ de solicitação no período pré-oficina e $3,7 \%$ no período pós oficina, na faixa etária de 40 a 49 foram registradas $36,7 \%$ de solicitação antes do período de realização das oficinas e 33,4\% posterior a estas, na faixa etária de 50 a 69, recomendada pelo Ministério da saúde para solicitação de mamografia de rastreamento população alvo foram solicitadas $54,5 \%$ no período anterior a capacitação e $59,4 \%$ após as oficinas, nas idades de 70 anos ou mais registra-se $3,4 \%$ das solicitações de mamografias anterior as oficinas e $3,2 \%$ posterior a estas (Gráfico 1 ).

Gráfico 1 - Comparação de Percentual de Mamografias de Rastreamento Por Idade no Período Pré e Pós Capacitação na Região de Integração do Xingu.

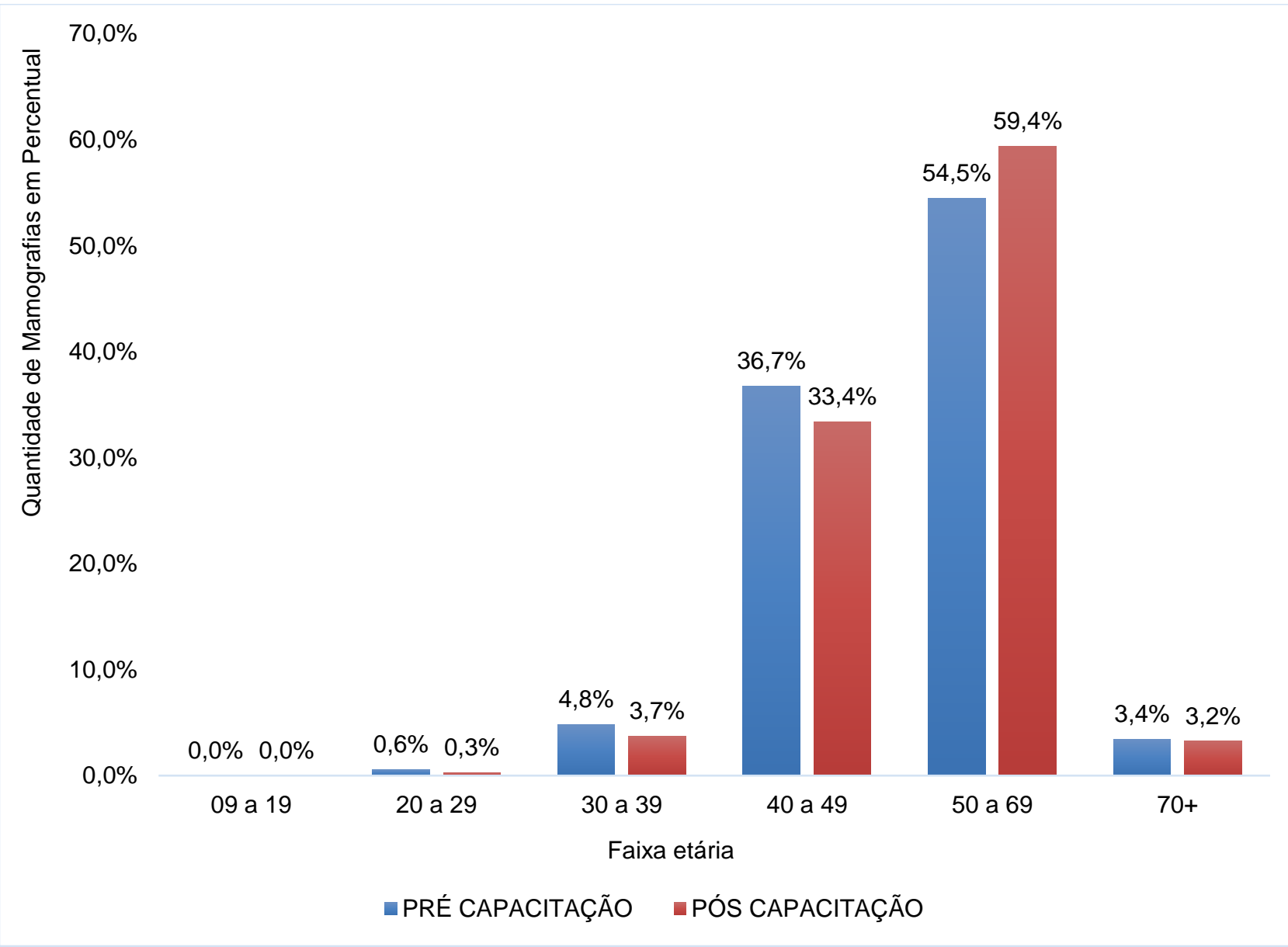

Fonte: Sobral RVS, et al., 2020; dados extraídos do Sistema de Informação do Câncer (SISCAN).

Traz-se o registro de solicitação de mamografias de rastreamento para população de alto risco, preconizadas pelo Ministério da Saúde para mulheres que possuem parentes de primeiro grau com diagnóstico ou óbito por câncer de mama e/ou câncer de ovário com idade maior que 35 anos.

Para este tipo de solicitação, levando-se em consideração a faixa etária de maior ou menor de 35 anos no período anterior e posterior às oficinas de atualização das diretrizes de rastreamento do câncer de mama, obteve-se o seguinte resultado: 31 mamografias solicitadas para população de alto risco anterior às oficinas, destas $1(3,2 \%)$ mamografia solicitada foi para uma pessoa menor de 35 anos e 30 (96,8\%) para maiores de 35 anos. No período posterior às oficinas, foram solicitadas um total de 40 mamografias, sendo que deste total $2(5 \%)$ mamografias solicitadas foram para menores de 35 anos e $38(95 \%)$ para maiores de 35 anos (Gráfico 2). 
Gráfico 2 - Mamografia De Rastreamento para população alvo solicitada nos municípios da Região Xingu.
40
35
30
25
20

15

10

5

0
30

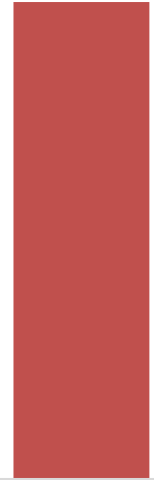

Pré Capacitação
38

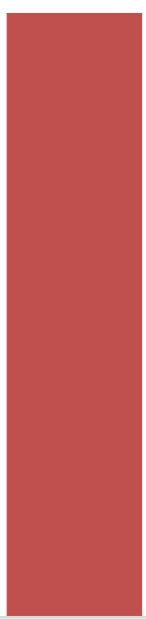

Pós-Capacitação

a $<35$ anos $\quad>35$ anos

Fonte: Sobral RVS, et al., 2020; dados extraídos do Sistema de Informação do Câncer (SISCAN).

Reflete-se as mamografias solicitadas para mulheres já tratadas de câncer de mama na região Xingu, anterior e posterior ao período de realização das oficinas, chegando-se ao seguinte resultados: Durante todo período de estudo foram solicitadas 34 mamografias de rastreamento para mulheres já tratadas de câncer de mama, sendo que 13 destas foram solicitadas no período anterior as oficinas e 21 no período após as oficinas (Gráfico 3).

Gráfico 3 - Mamografia de Rastreamento para mulheres já tratadas por câncer de mama solicitadas nos municípios da Região Xingu.

25

21

20

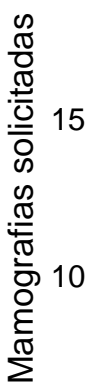

13

5

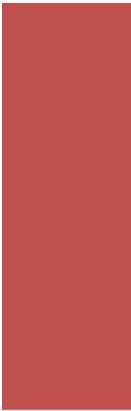

Pré Capacitação

Pós-Capacitação

Fonte: Sobral RVS, et al., 2020; dados extraídos do Sistema de Informação do Câncer (SISCAN). 
Demonstra-se o quantitativo de mamografias de diagnóstico solicitadas pelos municípios da Região Xingu, as quais referem-se ao tipo de mamografias solicitadas quando há suspeita clínica de alterações sugestivas de câncer de mama. Para este tipo de solicitação encontrou-se o resultado total, no período de estudo, de 20 mamografias de diagnóstico solicitadas, destas, 8 (40\%) solicitadas no período que antecedeu as oficinas e $12(60 \%)$ no período posterior a realização das oficinas (Gráfico 4).

Gráfico 4 - Mamografia de Diagnóstico solicitadas nos municípios da Região Xingu.

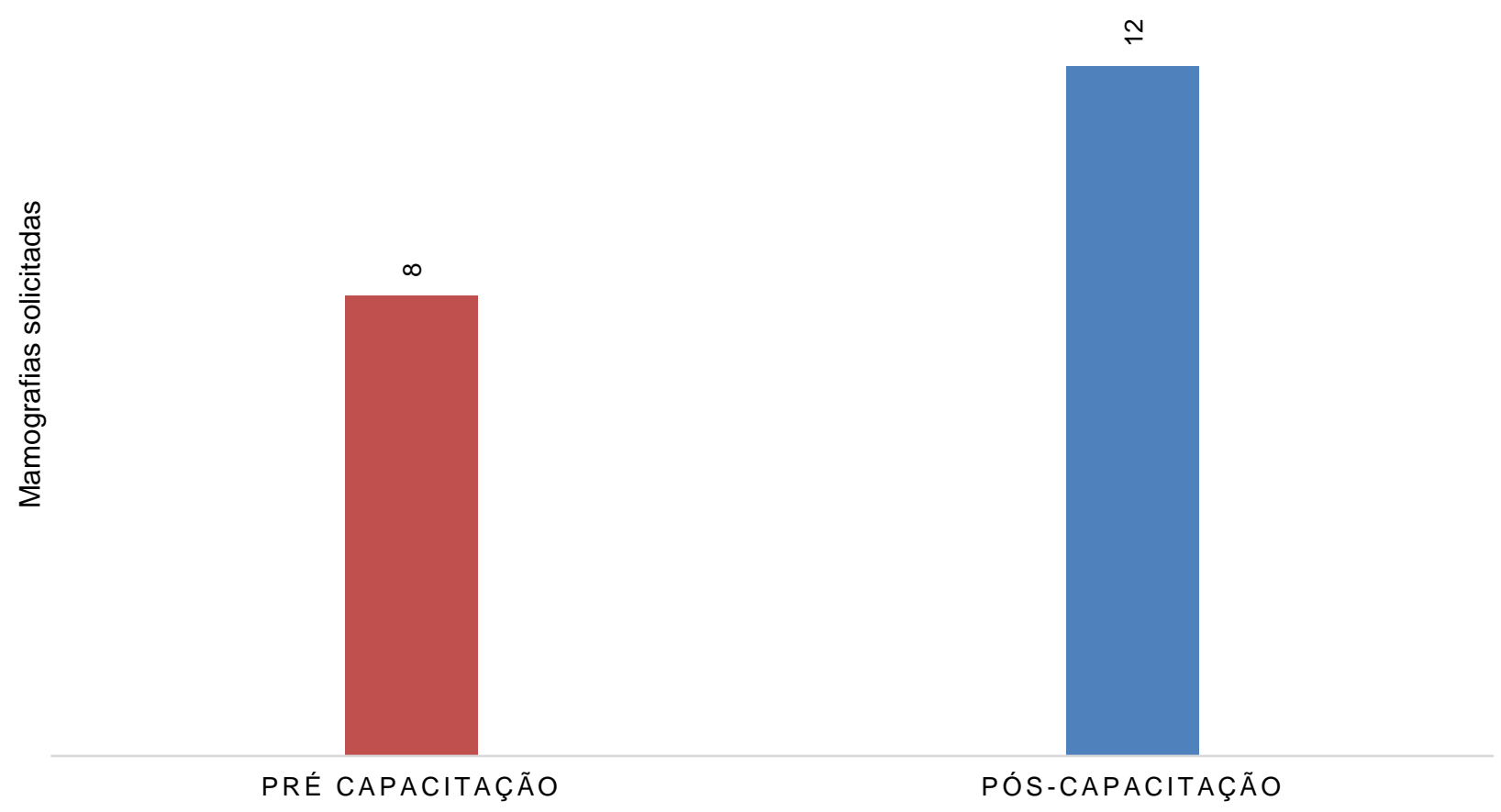

Fonte: Sobral RVS, et al., 2020; dados extraídos do Sistema de Informação do Câncer (SISCAN).

\section{DISCUSSÃo}

As capacitações em educação em saúde aos profissionais médicos, enfermeiros e da regulação, quanto à indicação de solicitação da mamografia de rastreio e diagnóstica, realizadas na Região de Integração de Xingu, permitiram uma atualização, aprendizagem e diferenciação dos protocolos preconizados pela Sociedade Brasileira de Mastologia e pelo INCA/Ministério da Saúde. Segundo Werneck M, et al. (2009), é de fundamental importância a existência dos protocolos em serviços de saúde tratando-se da rede pública, pois favorece a construção de um modelo de trabalho de atenção em saúde.

A mamografia de rastreio relacionada à idade apresentou como um todo uma diminuição percentual quanto à solicitação de MMG de 09 a 50 anos, bem como acima de 70 anos. Nota-se que a construção desta variável levou em consideração o que é preconizado como estratégia de rastreamento pelo INCA segundo a faixa etária (INCA, 2011). Apesar de conseguirmos uma diminuição neste percentual, ainda observamos solicitações equivocadas por estes profissionais quanto ao fator idade e indicação de solicitação. Isto somente resolver-se-á diante da continuidade da Educação Permanente em Saúde (EPS), visto que foi realizado somente um encontro em cada município de abrangência da região Xingu. Necessitando, portanto, de uma periodicidade e uma prática institucionalizada diante destes profissionais.

Consoante Carotta F, et al. (2009), a EPS ajuda aprimorar as práticas de serviços da equipe multiprofissional, tornando-os mais participativos, interessados, proporcionando a valorização de seus conhecimentos, aumentando, assim, oportunidades de aprendizagem no local de trabalho. Bem como, a arte 
da educação em saúde é o campo prático a qual proporciona vínculos entre a ação academicista médica e o saber popular (VASCONCELOS E, 2001). Ademais, a promoção da EPS colabora no desenvolver de cada passo da metodologia da problematização, baseada em 5 etapas, denominada de "Arco de Charles Maguerez": observação da realidade, identificação dos pontos-chave, teorização sobre a situação observada, geração de hipóteses e aplicação de soluções (BERBEL N, 2012).

Conforme a Sociedade Brasileira de Mastologia (SBM) e Urban LABD, et al. (2017), recomenda-se MMG de rastreio entre as faixas 40 a 74 anos. Por conseguinte, foi acordado com os profissionais médicos, os quais compactuam com as diretrizes da SBM, que estes estariam aptos realizar a solicitação de MMG de rastreio a partir dos 40 anos até os 74 anos. Com os demais profissionais enfermeiros, ratificamos a solicitação de MMG somente na faixa etária preconizada pelo INCA/MS de 50 a 69 anos de idade. Conforme os resultados, $4 \%$ das solicitações (42 solicitações) caracterizam-se como não assertivas, estando tanto abaixo da faixa etária preconizada pela SBM (40 a 74 anos) quanto abaixo da faixa etária preconizada pelo MS (50 a 69 anos). Estimamos que este percentual é decorrente de uma alta rotatividade de profissionais médicos da região, assim como da não participaram da capacitação.

Outro fator de risco importante, e crucial indagação durante a anamnese, é o histórico familiar da paciente. Coughlin SS e Cypel Y (2019) afirmam que possuir um histórico familiar positivo para câncer de mama é um fator de risco estabelecido à doença, onde mulheres com familiares de primeiro grau para esta mácula possuem risco em torno de duas vezes maior de desenvolver esta patologia. Por conseguinte, é indicado tanto pela SBM quanto pelo INCA/MS, o inicio do rastreamento com a solicitação de mamografia para mulheres com esse perfil a partir dos 35 anos. Em decorrência disto, de acordo com os resultados encontrados, no período de pós capacitação os profissionais da Região de Integração do Xingu tiveram 95\% de assertiva quanto à solicitação. Acreditamos que os $5 \%$ destas solicitações (2 solicitações), decorrente do município de Porto de Moz (PA), sejam de médicos rotativos os quais não receberam nosso treinamento.

Durante as capacitações, defendeu-se a importância de reconhecer a mamografia diagnóstica, bem como diferenciá-la da mamografia de rastreio. É importante destacar que a estratégia de diagnóstico precoce colabora à diminuição do estágio de apresentação da doença (WHO, 2007). Esta mesma estratégia ressalta o papel do aprendizado dos profissionais da saúde em conjunto com a educação da mulher diante dos sinais e sintomas os quais levam para o reconhecimento do câncer de mama. Assim, de acordo com as diretrizes para a detecção precoce do câncer de mama no Brasil, recomenda-se como sinais para mamografia diagnóstica:

"Qualquer nódulo mamário em mulheres com mais de 50 anos; nódulo mamário em mulheres com mais de 30 anos, que perdure para além de um ciclo menstrual; nódulo mamário de consistência endurecida e fixo ou que esteja aumentando de tamanho, em mulheres adultas de qualquer faixa etária; descarga papilar sanguinolenta unilateral; lesão eczematosa da pele que não responde a tratamentos tópicos; presença de linfadenopatia axilar; aumento progressivo do tamanho da mama com a presença de sinais de edema, como pele com aspecto de casca de laranja; retração na pele da mama; mudança no formato do mamilo" (BRASIL, 2015).

Em decorrência disto, nota-se uma disparidade diante mamografia de rastreamento e diagnóstica, visto que na Região de Integração do Xingu obteve-se 1.108 solicitações para mamografia de rastreio diante de 12 solicitações para mamografia diagnóstica, mesmo posterior as oficinas realizadas; esta última correspondendo a 1\%. Este baixo percentual perceptivelmente nos acusa dois cruciais pontos. O primeiro: a importância de nossos profissionais reconhecerem achados clínicos, por meio de um bom exame físico (ectoscopia), sendo este direcionados aos sinais e sintomas mastológicos. Dentre estes, o sinal com maior probabilidade de desencadear câncer de mama é o nódulo mamário, o qual se faz presente em $90 \%$ dos casos confirmados posteriormente como câncer (MCCOWAN C, et al., 2011).

Conforme Silva C, et al. (2009), é fato que o exame físico contribui para construção clínica, onde as evidências obtidas por intermédio das técnicas semiológicas de inspeção, palpação, percussão e ausculta ajudarão no bom diagnóstico. O segundo ponto é a necessidade de não sobrecarregar o Sistema Único de 
Saúde (SUS) com solicitações errôneas quando estas deveriam ser diagnósticas e não por rastreamento. Resultando, portanto, em um atraso para confirmação patológica à paciente (FIGUEIREDO M, et al., 2017). Em concordância com Barber MD, et al. (2004), tardar o no diagnóstico o início do tratamento é associado à menor sobrevida, visto que um tempo superior a três meses entre o surgimento dos sintomas e o início do tratamento reduz a taxa de sobrevivência em até $12 \%$.

Por fim, como observado nos resultados, em 2020 é explícito que a demanda de solicitações pós capacitação diminuíra devido ao reflexo do impacto da pandemia do novo coronavírus. Sabendo que o Hospital de referência para realização deste exame na região, Hospital Regional Público da Transamazônica (HRPT), localizado no município de Altamira-PA, paralisou a oferta do exame de mamografia entre os meses de março-setembro de 2020. Visto que esta paralização causou transtornos às pacientes que dependem do diagnóstico da doença, principalmente àquelas que são referenciadas dos municípios que integram a microrregião, pois são pessoas as quais moram distantes do município de Altamira.

De acordo com SBCO (2020), desde o início da pandemia de Covid-19, em torno 50 mil brasileiros deixaram de ser diagnosticados com câncer por não irem ao atendimento especializado. É válido destacar também que, segundo Sheng JY (2020), o período pandêmico por COVID-19 prejudicou os aspectos da prática clínica, sendo estes: atendimento de urgência e emergência, paciente eletivos e ambulatoriais, enfermarias, assim como conferências em saúde, ensino e pesquisa.

\section{CONCLUSÃO}

Estima-se que o objetivo de "avaliar o rastreamento de Câncer de Mama por meio da solicitação de mamografia" trouxe satisfação à equipe, visto que pudemos mudar a realidade das solicitações por meio da capacitação dos profissionais de saúde. Ademais, os propósitos deste projeto serviram para mostrar que os resultados esperados foram bons e geraram efeitos. Contudo, estes ainda foram insuficientes, reforçando, portanto, a necessidade de uma educação permanente aos profissionais da Região de estudo sempre com base Diretrizes Brasileiras de Rastreamento do Câncer de mama. Bem como, é importe à equipe multiprofissional possuir domínio diante da solicitação da mamografia de rastreio e diagnóstica, sabendo diferenciar e aplicá-las com intuito de maiores benefícios à principal protagonista: a mulher.

\section{REFERÊNCIAS}

1. BARBER MD, et al. Diagnostic delay in breast cancer. British Journal of Surgery 2004; 91: 49-53.

2. BERTELLI AP. Iconografia das mamas humanas. Iconography of humans breasts, An. paul. med. Cir, 1990;117(3/4): 41-69.

3. BERBEL N. Metodologia da problematização com o arco de Maguerez: uma reflexão teórico-epistemológica. Londrina: Eduel, 2012b. 204p

4. BRASIL. Ministério da Saúde. Secretaria de Atenção à Saúde. Departamento de Atenção Básica. Controle dos cânceres do colo do útero e da mama / Ministério da Saúde, Secretaria de Atenção à Saúde, Departamento de Atenção Básica. - 2. ed. - Brasília: Editora do Ministério da Saúde, 2013. 124 p.: il.

5. BRASIL. Ministério da Saúde. Secretaria de Gestão do Trabalho e da Educação na Saúde. Departamento de Gestão da Educação na Saúde. Curso de atualização em mamografia para técnicos e tecnólogos em radiologia / Ministério da Saúde, Secretaria de Gestão do Trabalho e da Educação na Saúde, Departamento de Gestão da Educação na Saúde. - Brasília: Ministério da Saúde, 2014. 186 p.: il.

6. BRASIL. Ministério da Saúde. Secretaria de Atenção à Saúde. Instituto Nacional do Câncer. Coordenação de Prevenção e Vigilância. Controle do Câncer de Mama - Documento do Consenso. 2014.

7. BRASIL. Ministério da Saúde, Instituto Nacional de Câncer José de Alencar Gomes da Silva. Estimativa 2020. Rio de Janeiro; 2019.

8. BRASIL. Ministério da Saúde, Instituto Nacional de Câncer José de Alencar Gomes da Silva. Diretrizes para a detecção precoce do câncer de mama no Brasil. Rio de Janeiro; 2015.

9. CAROTTA F, et al. Educação permanente em saúde: uma estratégia de gestão para pensar, refletir e construir práticas educativas e processos de trabalhos. Saúde e Sociedade; 2009;18:48-51.

10. COUGHLIN SS, CYPEL Y. Epidemiology of Breast Cancer in Women. Adv Exp Med Biol. 2019; 1152:9-29.

11. FERLAY J, et al. Cancer incidence and mortality worldwide: Sources, methods and major patterns in GLOBOCAN 2012. França, Int. J. Cancer, 2014; 136: E359-E386.

12. FERLAY J, et al. Estimating the global cancer incidence and mortality in 2018: GLOBOCAN sources and methods. França, Int. J. Cancer, 2019; 144:1941-1953. 
13. FIGUEIREDO M, et al. Solicitação de exames de apoio diagnóstico por médicos na Atenção Primária à Saúde. Saúde em Debate, vol. 41, no 114 p. 729-40.

14. GARNIER HS, COLONNA M. Épidémiologie des cancers du sein. La Presse Médicale, 2019; 48:1076-1084.

15. INCA. Instituto Nacional de Câncer José Alencar Gomes da Silva. Estimativa 2016: incidência de câncer no Brasil. Rio de Janeiro: Instituto Nacional de Câncer José Alencar Gomes da Silva; 2015.

16. INCA. Instituto Nacional De Câncer José Alencar Gomes Da Silva. Programa Nacional de Controle do Câncer de Mama. Rio de Janeiro; 2011.

17. JESINGER RA. Breast anatomy for the interventionalist. Tech Vasc Interv Radiol. 2014 Mar;17(1):3-9.

18. MCCOWAN C, et al. Identifying suspected breast cancer: development and validation of a clinical prediction rule. Br J Gen Pract. 2011 May;61(586):e205-14.

19. NETO ML, SANTANA L. 2020. In: SOCIEDADE BRASILEIRA DE CIRURGIA ONCOLÓGICA: SENSU Consultoria de Comunicação. Disponível em: https://sbco.org.br/2020/05/14/sociedades-medicas-apontam-reducao-de-70-dascirurgias-e-que-50-mil-brasileiros-nao-receberam-diagnostico-de-cancer/. Acesso em: 21 out. 2020.

20. SBCO. 2020. In: "Sociedades médicas apontam redução de $70 \%$ das cirurgias e que 50 mil brasileiros não receberam diagnóstico de câncer". Disponível em: https://sbco.org.br/2020/05/14/sociedades-medicas-apontam-reducao-de-70das-cirurgias-e-que-50-mil-brasileiros-nao-receberam-diagnostico-de-cancer/. Acesso em: 02 jan. 2021.

21. SHENG JY, et al. Management of Breast Cancer During the COVID-19 Pandemic: A Stage- and Subtype-Specific Approach. JCO Oncol Pract. 2020 Oct;16(10):665-674.

22. SILVA C, et al. O ensino do exame físico em suas dimensões técnicas e subjetivas. Texto \& Contexto - Enfermagem, vol. 18, núm. 3, julio-septiembre, 2009, pp. 458-465.

23. VASCONCELOS E. Redefinindo as práticas de Saúde a partir de experiências de Educação Popular nos serviços de saúde. Interface - Comunicação, Saúde, Educação, vol. 5, no 8, fevereiro de 2001, p. 121-26.

24. WHO. WORLD HEALTH ORGANIZATION. 2007. IN: Cancer control: knowledge into action: WHO guide for effective programmes. Disponível em: https://apps.who.int/iris/bitstream/handle/10665/43743/9241547338_eng.pd. Acesso em: 02 jan. 2021

25. WERNECK M, et al. Protocolos de cuidados à saúde e de organização do serviço. Nescon UFMG, $2009,88$. 